\title{
APLICACIÓN DE LAS TÉCNICAS NO DESTRUCTIVAS PULL-OFF Y ULTRASONIDOS EN EL CONTROL DE CALIDAD DEL REFUERZO CON MATERIALES COMPUESTOS EN ESTRUCTURAS DE CONCRETO
}

\author{
APPLICATION OF NON-DESTRUCTIVE PULL-OFF \\ AND ULTRASONIC TECHNIQUES IN REINFORCEMENT \\ QUALITY CONTROL WITH COMPOSITE MATERIALS \\ IN CONCRETE STRUCTURES
}

Victoriano González Fernández*

Ángela Barrios-Padura"

Marta Molina-Huelva"**

Fecha de recepción: 28 de noviembre de 2016

Fecha de revisión: 19 de abril de 2017

Fecha de aprobación: 20 de junio de 2017

Cómo citar: V. González Fernández, A. Barrios-Padura y M. Molina-Huelva, "Aplicaciones de las técnicas no destructivas Pull-Off y ultrasonidos en el control de calidad del refuerzo con materiales compuestos en estructuras de concreto," Ciencia e Ingeniería Neogranadina, vol. 28, no. 1, pp. 5-26. DOI: http://dx.doi.org/10.18359/rcin.2593

\section{RESUMEN}

El sistema de refuerzo de estructuras de concreto mediante materiales compuestos se emplea de forma intensiva desde hace 50 años. Sin embargo, aún son numerosas las incógnitas respecto a su comportamiento a largo plazo, por lo cual es fundamental el control de la unión

\footnotetext{
* Ingeniero de la Edificación y máster en Arquitectura y Patrimonio Histórico. Director ténico, Labrum Diagnosis y Asesoramiento Especializado S.L., Bollullos de la Mitación, Sevilla, España. Correo electrónico: victorianogonzalez@labrum.eu. ORCID: http://orcid.org/0000-0003-0560-1297

** Arquitecta y doctora en Arquitectura. Profesora titular, Universidad de Sevilla, Escuela Técnica Superior de Arquitectura de Sevilla (ETSA), Departamento de Construcciones Arquitectónicas I, Sevilla, España. Correo electrónico: abarrios@us.es. ORCID: http://orcid.org/0000-0003-1054-010X

*** Arquitecta y Doctora en Arquitectura. Profesora, Universidad de Sevilla, España. Escuela Técnica Superior de Arquitectura de Sevilla (ETSA), Departamento de Estructuras de Edificación e Ingeniería del Terreno, Sevilla, España. Correo electrónico: martamolina@us.es. ORCID: http://orcid.org/0000-0002-6402-2327
} 
entre el concreto y el refuerzo para asegurar su calidad. Es un sistema de refuerzo que se realiza por adhesión, y como consecuencia de la incertidumbre sobre la tensión de adherencia, en la práctica se resuelve con anclajes que encarecen la obra y que resultan inviables en grandes superficies de refuerzo. Una correcta puesta en obra y con garantías del adecuado comportamiento del refuerzo optimiza y valoriza este sistema. La implantación de un plan de control de calidad apropiado garantiza la optimización del refuerzo y permite establecer los criterios de mantenimiento adecuados. En esta investigación se ha desarrollado un plan experimental para el control de calidad de la puesta en obra, basado en el estudio de la unión entre concreto y las bandas de refuerzo, teniendo en cuenta diferentes tipos de concreto y sistemas de preparación superficial. Un plan de control de calidad in situ con ensayos semidestructivos tipo pull-off y no destructivos tipo ultrasonidos permite verificar la efectividad del refuerzo.

Palabras clave: materiales compuestos, refuerzo de estructuras, control de calidad, adherencia, ensayos pull-off, ultrasonidos.

\section{ABSTRACT}

The reinforcement system of concrete structures using composite materials has been widely used for more than fifty years. However, there are still many unknowns regarding their longterm behavior, as the control of the bond between the concrete and the reinforcement materials being essential to ensure their quality. It is a reinforcement system that is made by adhesion, and as consequence of the uncertainty on the adhesion tension, in practice, it is solved with anchorages that make the work more expensive and which are not feasible for large areas of reinforcement. A correct installation that guarantees the proper behavior of the reinforcement materials would optimize this type of strengthening system and its applicability. The implementation of an appropriate quality control plan guarantees the optimization of the system and allows to establish the proper maintenance criteria. This research presents an experimental program for the quality control of laying, based on the study of the bond between the concrete and fiber composite sheets, taking into account different types of concrete and surface preparation techniques. An in situ quality control plan with semi-destructive pull off and non-destructive ultrasonic type tests allows verifying the effectiveness of the reinforcement.

Keywords: Composite materials, structural strengthening, quality control, bond, pull off test, ultrasonic test.

\section{INTRODUCCIÓN}

Una estructura de concreto armado debe satisfacer durante su vida útil los requisitos para los que fue diseñada. Sin embargo, sus condiciones pueden cambiar a lo largo de su vida útil por lo que, pasado cierto tiempo, la estructura puede precisar operaciones de acondicionamiento y refuerzo que le permitan hacer frente a la nueva situación de ser- 
vicio. Uno de los sistemas más eficaces para estas operaciones es el refuerzo mediante materiales compuestos y estratégicos para el desarrollo sostenible [1]. Se define como material compuesto a "todo material combinado a partir de una unión (no química) de dos o más componentes, que dan lugar a propiedades características específicas, no siendo ninguna de las anteriores" [2]. En la actualidad, una solución muy utilizada son los elementos estructurales de concreto armado (Fibre Reinforced Polymer-FRP), los cuales presentan indudables ventajas reconocidas en el ámbito profesional, pero también algunas desventajas que en ocasiones hacen dudar de su eficacia [3]. Uno de los aspectos positivos de emplear materiales compuestos como elementos de refuerzo se basa en sus excelentes propiedades mecánicas y en su anisotropía. El refuerzo puede ser ajustado a voluntad, orientando las fibras de acuerdo con las direcciones en las que se originan los mayores esfuerzos, lo que permite optimizar el material y reducir los costos.

Dos cuestiones de suma importancia en la ejecución del refuerzo son la calidad y el estado del soporte, y la adecuada colocación de la fibra. El conocimiento en mayor profundidad de las condiciones del soporte y del estado de la adherencia entre el concreto y los polímeros armados con fibra optimiza el refuerzo. La estructura y las propiedades de las uniones entre materiales, por una parte fibra-matriz y, por otra, FRP con el concreto, juegan un papel muy importante en las propiedades físicas y mecánicas finales del refuerzo. Los ensayos semidestructivos tipo pull-off [4] aportan suficiente información a este respecto, como se muestra en el presente trabajo. En 1997 Horiguchi y Saeki [5] realizaron estudios sobre la idoneidad de los ensayos y la influencia de la calidad del concreto en probetas reforzadas con laminados de fibra de carbono. Así, analizaron tres tipos de ensayos: de corte, de flexión y de tracción directa pull-off. Allen y Atadero [6] emplearon también el ensayo de pull-off para valorar el comportamiento del FRP a largo plazo.

Bai, Basheer, Cleland y Long [7] desarroIlan la historia del ensayo de pull-off y su aplicación en ingeniería. Es el único ensayo de adherencia sobre el concreto que está normalizado. Los documentos ASTM D7522 [8] y $\mathrm{ACl} 440$ 3R-12 en su parte 3 [6] proporcionan especificaciones detalladas. Asimismo, el documento de la Sociedad Japonesa de Ingenieros Civiles JSCE-E545-2000 muestra detalladamente el procedimiento para este tipo de ensayo [9]. Los ensayos de adhesión directa sobre el concreto se encuentran definidos en la norma europea UNE EN 1542 [10].

En cuanto a la técnica de ultrasonidos, Scarponi y Briotti [11], y Bastianini, Di Tommaso y Pascale [12] han utilizado ensayos de ultrasonidos para determinar defectos de adhesión. Por su parte, Ekenel y Myers [13], y Helmerich, Milmann y Wöstmann [14] utilizaron esta técnica de inspección no destructiva para la evaluación de delaminaciones en varios materiales compuestos. Por lo tanto, el control del comportamiento de la unión entre materiales es fundamental para eliminar la incertidumbre de la eficiencia de los refuerzos con materiales compuestos [15]-[16].

El objetivo de la investigación es evaluar la adherencia del refuerzo de FRP en estructuras de concreto armado mediante técnicas no destructivas y semidestructivas, 
como parte de un protocolo de control de calidad del refuerzo que permita valorar su eficacia. Para la valoración de la efectividad del enlace entre el sustrato y el refuerzo se distinguen dos escenarios: uno local, referido al sustrato por reforzar, y otro de conjunto, que contempla el sustrato y el refuerzo. La singularidad de la investigación reside en el estudio de un método no destructivo, como es el ensayo de ultrasonidos, como prueba válida de control de calidad, basándose en la unión entre el refuerzo de FRP y el soporte [17]. Esta es una técnica de fácil empleo en obra, en zonas de difícil acceso y de rápida ejecución, a partir de la cual se han podido establecer correlaciones cuantificables con otras técnicas semidestructivas como son las tipo pull-off y destructivas como los ensayos a flexión.

\section{MATERIALES Y MÉTODOS}

Para la ejecución de la investigación completa e implantación del plan de ensayos se elaboraron 100 probetas con dos tipos de concreto, de una resistencia 25 y $40 \mathrm{MPa}, 50$ de cada tipo. El primero representa a la clase más comunmente empleada en edificación y el segundo, en infraestructuras y estructuras singulares.

Para el desarrollo del proyecto de investigación se confeccionaron 30 probetas armadas (15 con concreto HA25 y 15 con concreto HA40), y 70 sin armar (35 con concreto HM25 y 15 con concreto HM40). Esta diferencia de distribución de probetas se dio por la distribución y el número de todos los tipo de ensayo programados, y se esablació de esta manera como lote mínimo por tipo de ensayo una cantidad de 5, y así resultó la cantidad indicada. Del total de probetas empleadas en el proyecto de investigación, para el estudio de este artículo y aplicar las técnicas de pull-off y ultrasonidos se utilizó un total de 38. Se aplicó, además, refuerzo de FRP al $90 \%$ de las muestras, y el resto quedó para la obtención de los parámetros iniciales de la investigación.

Para las probetas armadas los cálculos se realizaron para la rotura a flexión de la sección en el dominio 2 , donde su resistencia a cortante es superior a su resistencia a flexión, y falla por falta de capacidad de su armadura traccionada. La armadura colocada ha consistido en $2 \varnothing 8 \mathrm{~mm}$ de acero $B$ $400 \mathrm{~S}$ colocadas en la parte inferior con recubirmiento inferior y lateral de $2,5 \mathrm{~cm}$ y $\sin$ armadura transversal.

El refuerzo se realizó con tejido de fibra de carbono tipo "SikaWrap". Este se colocó en toda la longitud de las probetas $(60 \mathrm{~cm})$, con el objetivo de que la longitud de anclaje fuese homogénea en todos los casos. Dicho tejido es de fibra de carbono unidireccional cosido y resina epoxi bicomponente comercial tipo "Sikadur-300", diseñada para la aplicación a los refuerzos de tejido de fibra de carbono.

Los ensayos realizados corresponden a dos grupos. El primero en el que se incluyen ensayos dirigidos a la determinación de propiedades intrínsecas (influencia de la superficie y de la estructura porosa del concreto en la adherencia) como los pull-off y ultrasonidos. El segundo determina las propiedades extrínsecas (solicitación mecánica), mediante ensayo mecánico a flexión. 
Ciencia e Ingeniería Neogranadina

\section{PROGRAMA EXPERIMENTAL}

Los ensayos para determinar la adherencia entre el concreto y el refuerzo se realizaron sobre una serie de probetas prismáticas de dimensiones $150 * 150 * 600 \mathrm{~mm}$, diseñadas dentro de un programa de investigación más amplio, al resultar estas las más versátiles para el conjunto de ensayos programados.

Las condiciones que se han tenido en cuenta para la ejecución de los ensayos y adhesión de las bandas de FRP han sido el tipo concreto y el acabo superficial de las probetas.

En cuanto al tipo de concreto, se estudiaron probetas de 25 y $40 \mathrm{MPa}$ de resistencia. Respecto al acabado superficial, previo a la aplicación de la fibra, se imprimieron tres clases de rugosidades diferentes en la superficie soporte de concreto. Así, la diferencia entre rugosidades, clasificadas como lisa-media-rugosa, se consigue según el procedimiento de preparación del soporte (Tabla 1).
Tabla 1. Tipo de tratamiento superficial del soporte

\begin{tabular}{|l|l|l|}
\hline $\begin{array}{c}\text { Código } \\
\text { superficie }\end{array}$ & \multicolumn{1}{|c|}{ Tratamiento } & Rugosidad \\
\hline A & Lijado & Lisa \\
\hline B & Cepillo de púas & Media \\
\hline C & Cepillo de desgaste & Rugosa \\
\hline \multicolumn{2}{|c|}{ Fuente: elaboración propia. } \\
\hline
\end{tabular}

En cada una de las probetas a las que se les colocó las bandas de FRP con resina, se emplearon tres caras para reproducir respectivamente las tres rugosidades. Se realizó un total de 120 ensayos tipo pull-off.

Puesto que durante el proceso de curado fue imprescindible ejercer presión, se diseñó un sistema con anillos metálicos formado por perfiles rectangulares tubulares que presionan el refuerzo y aseguran así una homogeneidad suficiente (Figs. 1-3). Desde los anillos metálicos a través de unos tornillos a los que se les da un par de apriete de 20 $\mathrm{N} / \mathrm{m}$, cada perfil presiona una banda de refuerzo sobre la probeta de concreto.

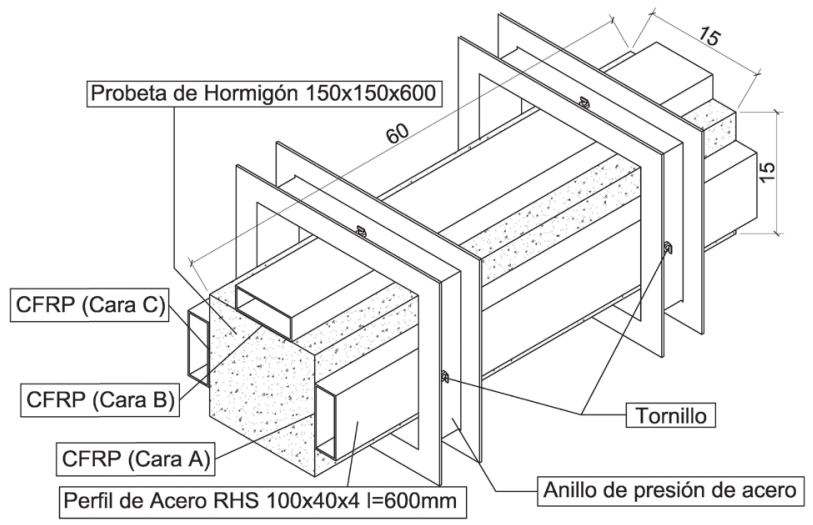

Fig. 1. Sistema empleado para la colocación del FRP y caras de tratamiento (cotas en cm). Fuente: elaboración propia. 


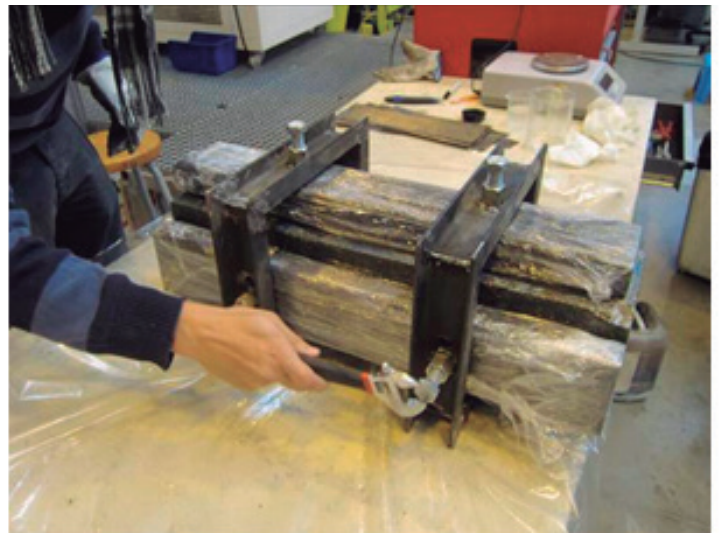

Fig. 2. Disposición de los anillos de presión Fuente: elaboración propia.

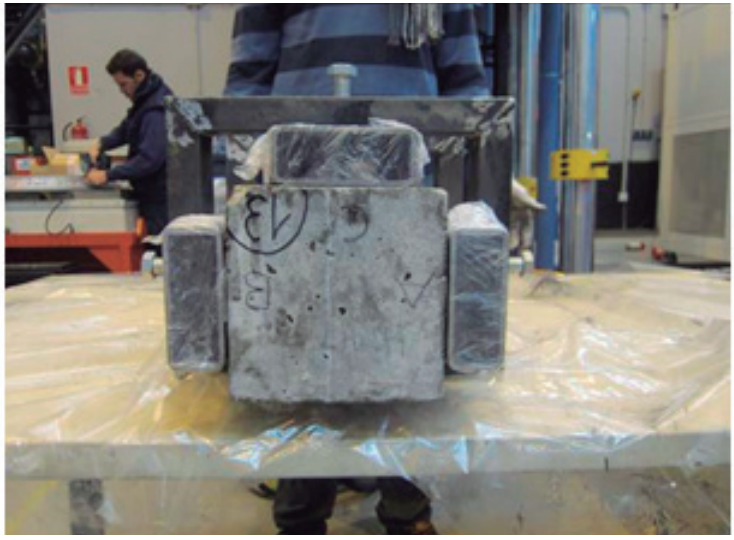

Fig. 3. Perfiles de presión sobre FRP Fuente: elaboración propia.

A continuación, se muestra un resumen del número de probetas utilizadas y número de ensayos ejecutados (Tabla 2):

Tabla 2. Conjunto de probetas ensayadas

\begin{tabular}{|c|c|c|c|c|c|}
\hline \multirow{2}{*}{$\begin{array}{c}N^{\circ} \text { DE } \\
\text { PROBETA }\end{array}$} & \multirow{2}{*}{ REF. PROBETA } & \multirow{2}{*}{$\begin{array}{l}\text { No ENSAYOS } \\
\text { FLEXIÓN }\end{array}$} & \multicolumn{2}{|c|}{$\begin{array}{l}N^{\circ} \text { DE ENSAYOS } \\
\text { ULTRASONIDOS }\end{array}$} & \multirow{2}{*}{$\begin{array}{c}\text { N}^{\circ} \text { ENSAYOS } \\
\text { PULL OFF }\end{array}$} \\
\hline & & & $\begin{array}{c}\text { SOBRE } \\
\text { CONCRETO }\end{array}$ & $\begin{array}{c}\text { SOBRE } \\
\text { FIBRA DE } \\
\text { CARBONO }\end{array}$ & \\
\hline 1 & (1) HM40-1A & 1 & 1 & 1 & * \\
\hline 2 & (2) $\mathrm{H} 40-1 \mathrm{~B}$ & 1 & 1 & 1 & * \\
\hline 3 & (3) $\mathrm{HM} 40-1 \mathrm{C}+\mathrm{FRP}$ & 1 & 1 & 1 & * \\
\hline 4 & (4) HM40-A +FRP + Espiga & 1 & 1 & 1 & * \\
\hline 5 & (5)HM40-B +FRP + Espiga & 1 & 1 & 1 & * \\
\hline 6 & (6) HM40-C +FRP + Espiga & 1 & 1 & 1 & * \\
\hline 7 & (7)HM40 (sin reforzar) & 1 & 1 & & \\
\hline 8 & (8) HM25-1A +FRP & 1 & 1 & 1 & * \\
\hline 9 & (9) HM25-1B +FRP & 1 & 1 & 1 & * \\
\hline 10 & (10)HM25-1C +FRP & 1 & 1 & 1 & * \\
\hline 11 & (11)HM25 (sin reforzar) & 1 & 1 & & \\
\hline 12 & (12) HM 40 +FRP (caras A,B,C) & & 1 & 1 & 15 \\
\hline
\end{tabular}


Ciencia e Ingeniería Neogranadina

\begin{tabular}{|c|c|c|c|c|c|}
\hline \multirow{2}{*}{$\begin{array}{c}\mathbf{N}^{\circ} \text { DE } \\
\text { PROBETA }\end{array}$} & \multirow[b]{2}{*}{ REF. PROBETA } & \multirow{2}{*}{$\begin{array}{l}\text { N }^{\circ} \text { ENSAYOS } \\
\text { FLEXIÓN }\end{array}$} & \multicolumn{2}{|c|}{$\begin{array}{l}N^{\circ} \text { DE ENSAYOS } \\
\text { ULTRASONIDOS }\end{array}$} & \multirow{2}{*}{$\begin{array}{l}\text { N}^{\circ} \text { ENSAYOS } \\
\text { PULL OFF }\end{array}$} \\
\hline & & & $\begin{array}{c}\text { SOBRE } \\
\text { CONCRETO }\end{array}$ & $\begin{array}{l}\text { SOBRE } \\
\text { FIBRA DE } \\
\text { CARBONO }\end{array}$ & \\
\hline 13 & (13) HM 40 +FRP (caras A,B,C) & & 1 & 1 & 15 \\
\hline 14 & (14) HM 25 +FRP (caras A,B,C) & & 1 & 1 & 15 \\
\hline 15 & (15)HA40-C+FRP & & 1 & 1 & $\star \star$ \\
\hline 16 & (16)HA40-C+FRP + Espiga & & 1 & 1 & 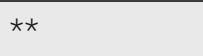 \\
\hline 17 & (17)HA40 (sin reforzar) & 1 & 1 & & \\
\hline 18 & (18)HA25-C+FRP & & 1 & 1 & * \\
\hline 19 & (19)HA25 (sin reforzar) & 1 & 1 & & \\
\hline 20 & (21) HA 40 - C & & 1 & 1 & * \\
\hline 21 & (22) HA 40 - C & & 1 & 1 & * \\
\hline 22 & (22) HM40-2A +FRP & 1 & 1 & 1 & * \\
\hline 23 & (23)HM40-2B +FRP & 1 & 1 & 1 & * \\
\hline 24 & (24)HM40-2C +FRP & 1 & 1 & 1 & * \\
\hline 25 & (25)HM25-2A +FRP & 1 & 1 & 1 & * \\
\hline 26 & (26)HM25-2B +FRP & 1 & 1 & 1 & * \\
\hline 27 & (27)HM25-2C +FRP & 1 & 1 & 1 & * \\
\hline 28 & (28) HM 40 +FRP (caras A,B,C) & & 1 & 1 & 15 \\
\hline 29 & (29) HM 25 +FRP (caras A,B,C) & & 1 & 1 & 15 \\
\hline 30 & (30)HM40-3A +FRP & 1 & 1 & 1 & * \\
\hline 31 & (31)HM40-3B +FRP & 1 & 1 & 1 & * \\
\hline 32 & (32)HM40-3C +FRP & 1 & 1 & 1 & * \\
\hline 33 & (33)HM25-3A +FRP & 1 & 1 & 1 & * \\
\hline 34 & (34)HM25-3B +FRP & 1 & 1 & 1 & * \\
\hline 35 & (35)HM25-3C +FRP & 1 & 1 & 1 & * \\
\hline 36 & (36) HM 40 +FRP (caras A,B,C) & & 1 & 1 & 15 \\
\hline 37 & (37) HM 25 +FRP (caras A,B,C) & & 1 & 1 & 15 \\
\hline 38 & (38) HM 25 +FRP (caras A,B,C) & & 1 & 1 & 15 \\
\hline
\end{tabular}

* No se realizan ensayos de pull-off, ya que alterarían el resultado de ensayo a flexión.

** Resultados no válidos.

Fuente: elaboración propia. 


\subsection{Descripción de los ensayos}

El ensayo de flexión se realizó mediante una prensa hidráulica de capacidad $1500 \mathrm{kN}$, según los criterios de la norma UNE-EN- 123905-2001 [18], la cual tiene una precisión de $1 \mathrm{~N}$. Los parámetros de ensayo fueron una velocidad de carga de $500 \mathrm{~N} / \mathrm{s}$, un intervalo de carga $140 \mathrm{~mm}$ y un intervalo soporte de $45 \mathrm{~mm}$.

El plan de ensayos a flexión del proyecto de investigación se realizó teniendo en cuenta la comparabilidad de los resultados en pro- betas sin reforzar con fibra de carbono, con el objeto de obtener un valor de referencia; para esto, se ensayaron 24 probetas para cada tipo de concreto, de las cuales, dado que el objetivo fue la adherencia del refuerzo, mayoritariamente se utilizaron de concreto en masa reforzadas distribuidas según se indica en la Tabla 3. Si bien, para el estudio que se expone acá se empleó sólo 25 probetas del total, 10 de cada tipo de concreto (HM25 y HM40), y el resto a efectos comparativos, 3 armadas y reforzadas, y 2 sin armar ni reforzar.

Tabla 3. Condiciones y variables en los ensayos de flexión

\begin{tabular}{|l|l|l|l|}
\hline \multicolumn{2}{|c|}{ Probetas de concreto en masa } & \multicolumn{2}{c|}{ Probetas de concreto armado } \\
\hline HM40 & HM25 & HA40 & HA25 \\
\hline $\begin{array}{l}1 \text { probeta } \\
\text { sin refuerzo FRP }\end{array}$ & $\begin{array}{l}\text { 1 probeta } \\
\text { sin refuerzo FRP }\end{array}$ & $\begin{array}{l}1 \text { probeta } \\
\text { sin refuerzo FRP }\end{array}$ & $\begin{array}{l}1 \text { probeta } \\
\text { sin refuerzo FRP }\end{array}$ \\
\hline 19 probetas reforzadas FRP & $\begin{array}{l}19 \text { probetas } \\
\text { reforzadas FRP }\end{array}$ & 3 probetas reforzadas FRP & 3 probetas reforzadas FRP \\
\hline
\end{tabular}

Fuente: elaboración propia.

El ensayo pull-off se realizó según la UNE EN 1542:2000 y consiste en la aplicación de una fuerza de tracción normal al plano de ensayo, con el objetivo de obtener la tensión de arrancamiento (MPa).

Se realizaron 120 ensayos sobre refuerzos de fibra de carbono aplicados a 8 probetas sin armar (4 probetas de cada tipo de concreto), en 3 de sus cuatro caras, y 5 ensayos por cara con diferentes tratamientos superficiales A, B, y C (Figs. 4-7). El resultado de ensayo, de cada cara, ha sido el de los 5 ensayos ejecutados en la misma. Los ensayos de ultrasonidos se realizaron según la UNE EN 12504-4:2006 [19], con el objetivo de estudiar la calidad de la aplicación del material compuesto. La posición de los palpadores se realizó mediante el sistema de transmisión indirecta de la probeta de concreto (Figs. 8 y 9). 


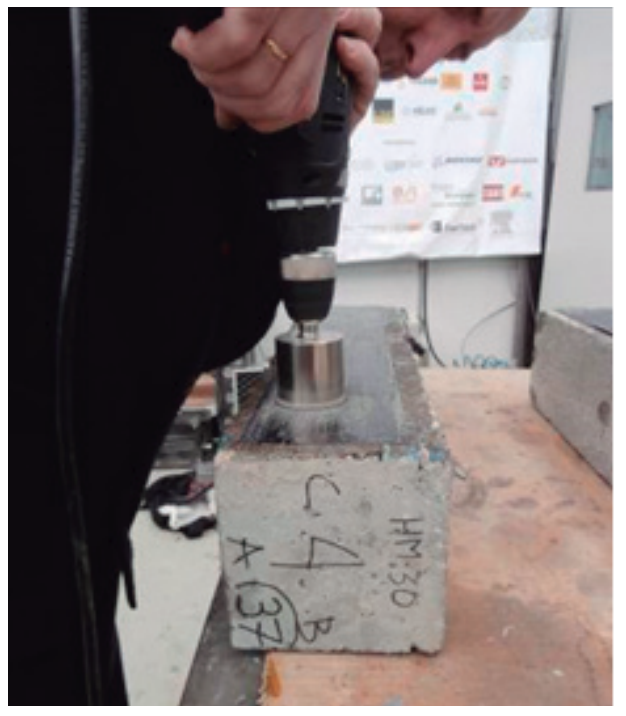

Fig. 4. Corte circular sobre FRP Fuente: elaboración propia.

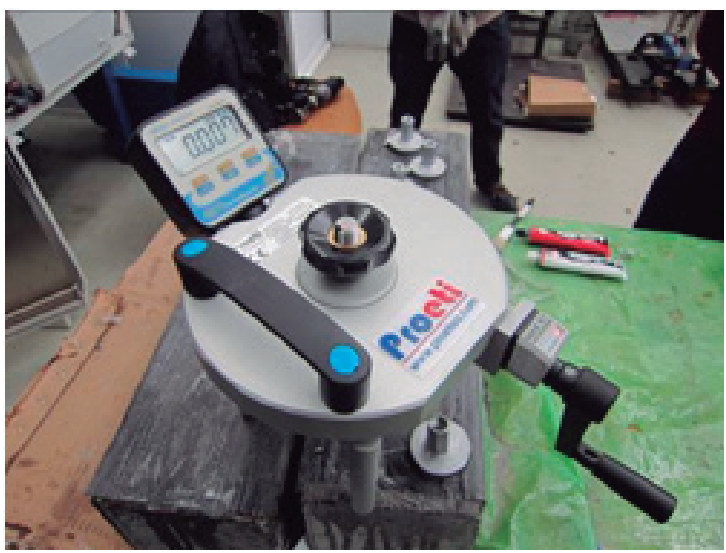

Fig. 6. Equipo para ensayo pull-off Fuente: elaboración propia.

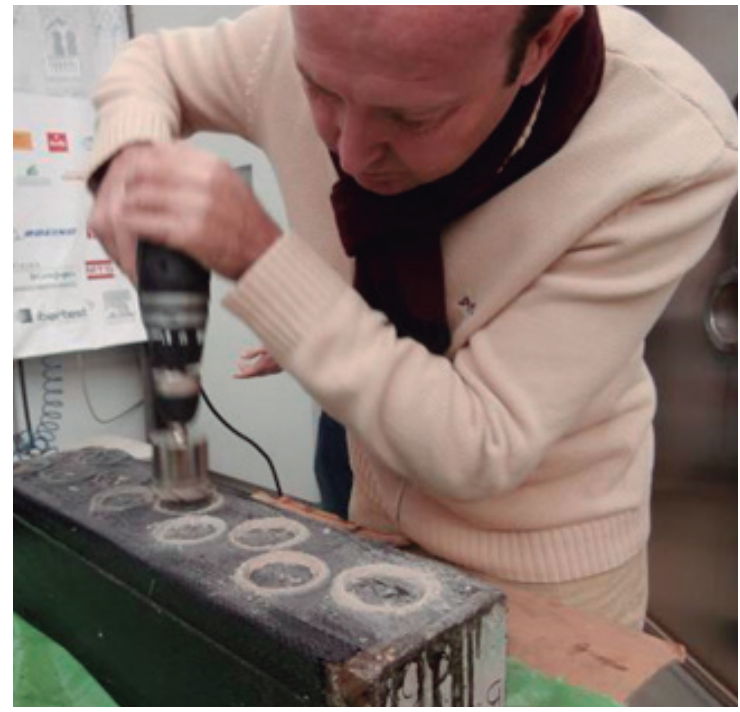

Fig. 5. Corte circular diferentes bandas de FRP Fuente: elaboración propia.

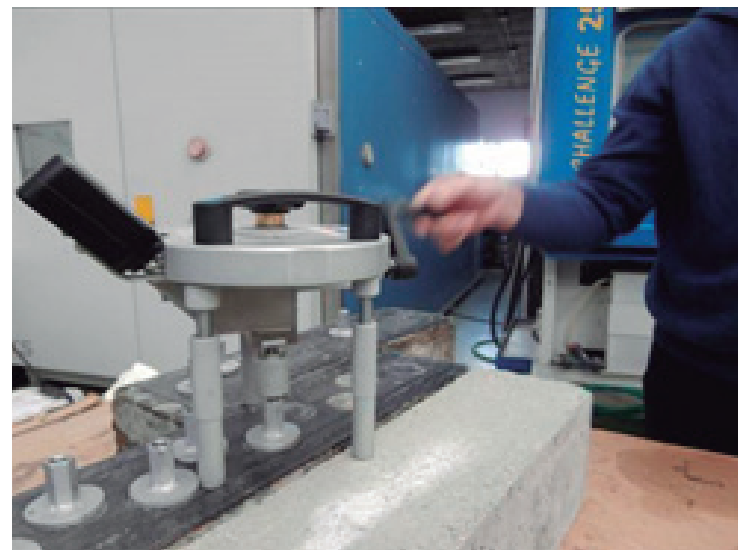

Fig. 7. Ejecución ensayo Pull-Off sobre la probeta Fuente: elaboración propia. 


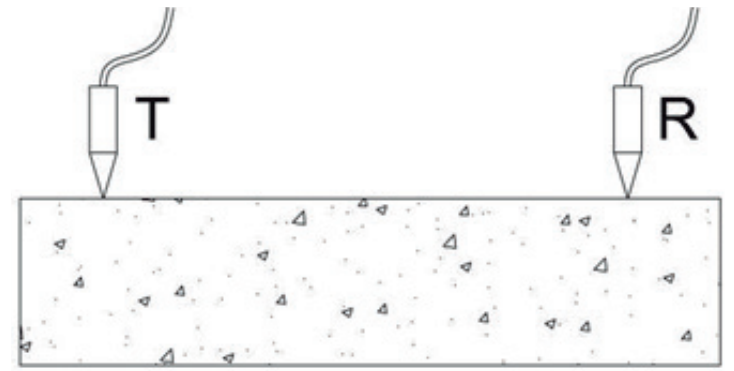

Fig. 8. Medición ultrasonidos método indirecto Fuente:Tomado de [19].

La toma de datos de ultrasonido se realizó sobre cada una de las 3 caras reforzadas de las 8 probetas destinadas a los ensayos de pull-off y sobre cada una de las caras de las 24 probetas destinadas para el ensayo a flexión. Se realizaron 72 medidas antes de la aplicación del refuerzo y otras 72 a posteriori una vez aplicado el refuerzo de FRP. Por lo tanto, como resultado se obtuvieron dos series de datos, una de los valores obtenidos sobre el concreto y otra sobre la fibra de carbono aplicada y endurecida.

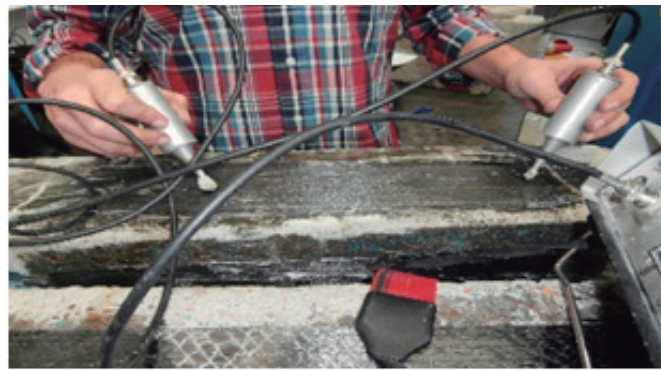

Fig. 9. Medición ultrasonidos método indirecto Fuente: elaboración propia.

\section{RESULTADOS Y DISCUSIÓN}

\subsection{Resultados de los ensayos tipo pull-off}

En la Tabla 4 y en la Fig. 10 se muestran los diferentes resultados obtenidos, en las cuales pueden verse los valores medios de la tensión de arrancamiento en MPa obtenidos en este ensayo, en relación con el tipo de concreto 25 o $40 \mathrm{MPa}$ y al tratamiento superficial A, B o C.

Tabla 4. Resultados de los ensayos de pull-off

\begin{tabular}{|l|l|l|}
\hline \multirow{2}{*}{ PROBETA } & \multicolumn{2}{c|}{ PULL-OFF } \\
\cline { 2 - 3 } & \multicolumn{1}{|c|}{$\begin{array}{c}\text { Tensión media de } \\
\text { arrancamiento (MPa) }\end{array}$} & \multicolumn{1}{c|}{$\begin{array}{c}\text { Porcentaje de rotura } \\
\text { en la unión }\end{array}$} \\
\hline (12) HM 40 - A+FRP & 5,33 & 14 \\
\hline (12) HM 40 - B+FRP & 3,24 & 93 \\
\hline (12) HM 40 - C+FRP & 2,13 & 25 \\
\hline (13) HM 40 - A+FRP & 3,23 & 60 \\
\hline
\end{tabular}


Ciencia e Ingeniería Neogranadina

\begin{tabular}{|c|c|c|}
\hline \multirow{2}{*}{ PROBETA } & \multicolumn{2}{|c|}{ PULL-OFF } \\
\hline & $\begin{array}{c}\text { Tensión media de } \\
\text { arrancamiento (MPa) }\end{array}$ & $\begin{array}{c}\text { Porcentaje de rotura } \\
\text { en la unión }\end{array}$ \\
\hline (13) HM 40 - B+FRP & 2,13 & 23 \\
\hline (13) HM 40 - C+FRP & 2,51 & 16 \\
\hline (14) HM 25- A+FRP & 2,51 & 1 \\
\hline (14) HM 25- B+FRP & 2,96 & 0 \\
\hline (14) HM 25- C+FRP & 3,23 & 10 \\
\hline (28) HM 40 - A+FRP & 2,56 & 2 \\
\hline (28) HM 40 - B+FRP & 2,02 & 50 \\
\hline (28) HM 40 - C+FRP & 1,52 & 29 \\
\hline (29) HM 25- A+FRP & 2,66 & 20 \\
\hline (29) HM 25- B+FRP & 1,07 & 29 \\
\hline (29) HM 25- C+FRP & 1,70 & 35 \\
\hline (36) HM 40 - A+FRP & 2,84 & 13 \\
\hline (36) HM 40 - B+FRP & 3,21 & 48 \\
\hline (36) HM 40 - C+FRP & 1,51 & 16 \\
\hline (37) HM 25- A+FRP & 1,78 & 24 \\
\hline (37) HM 25- B+FRP & 1,97 & 14 \\
\hline (37) HM 25- C+FRP & 2,92 & 0 \\
\hline (38) HM 25- A+FRP & 2,35 & 20 \\
\hline (38) HM 25- B+FRP & 2,00 & 18 \\
\hline (38) HM 25- C+FRP & 2,62 & 5 \\
\hline
\end{tabular}

Fuente: elaboración propia. 


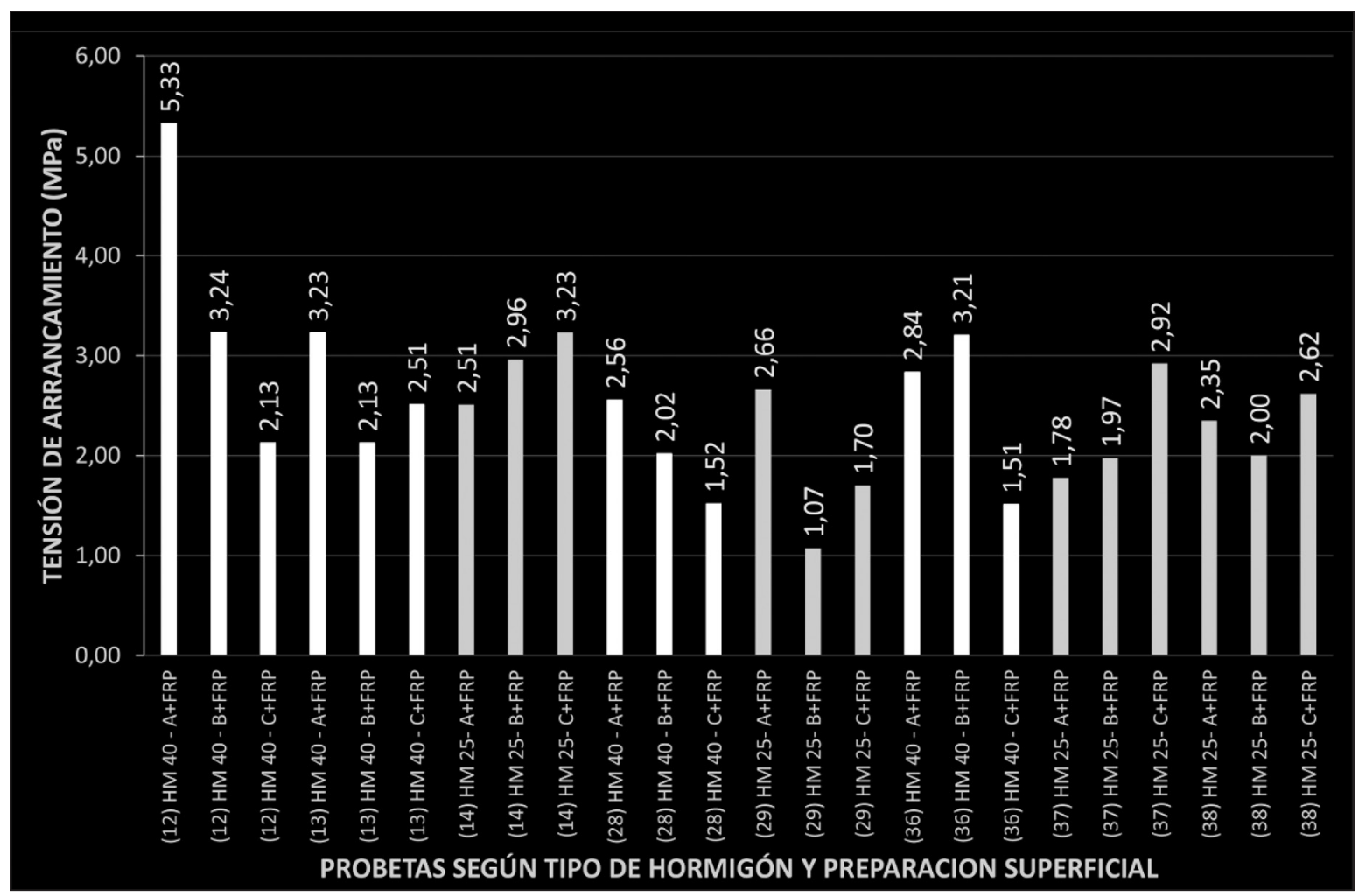

Fig. 10. Ensayos pull-off

Fuente: elaboración propia.

Consiguiendo la media de los valores obtenidos de la tensión de arrancamiento para cada tipo de concreto y tratamiento superficial, en la Fig. 11 se observa que la rugosidad influye en la tensión de arrancamiento.

En probetas de concreto HM-40, a medida que aumenta la rugosidad de la superficie disminuye la tensión de adherencia. En cuanto al tipo de concreto HM-25, en este no influye la preparación del soporte.

\subsection{Resultados ensayos de ultrasonidos}

En las Figs. 12 y 13 se muestran los resultados logrados en función del tipo de concreto HM-25 - HM-40 MPa y de la preparación del soporte A, $B$ o $C$, antes y después de colocar el refuerzo. 


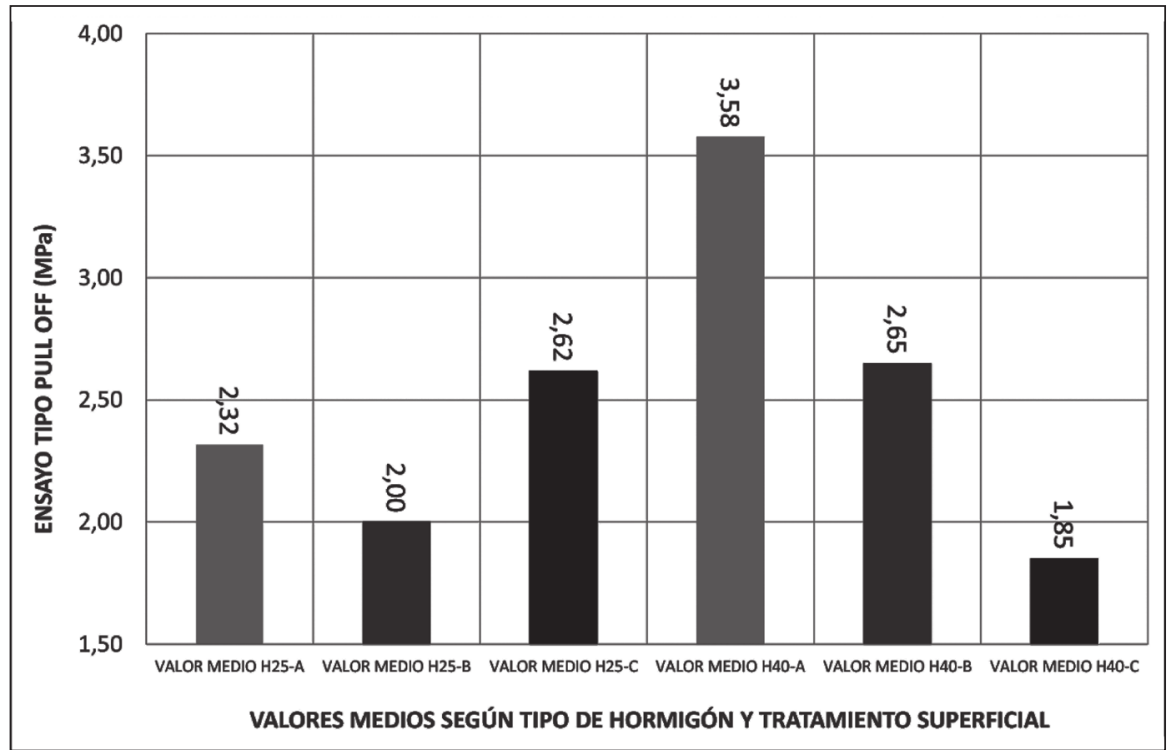

Fig. 11. Tensión de arrancamiento media del ensayo tipo pull-off según tratamiento superficial y tipo de concreto

Fuente: elaboración propia.

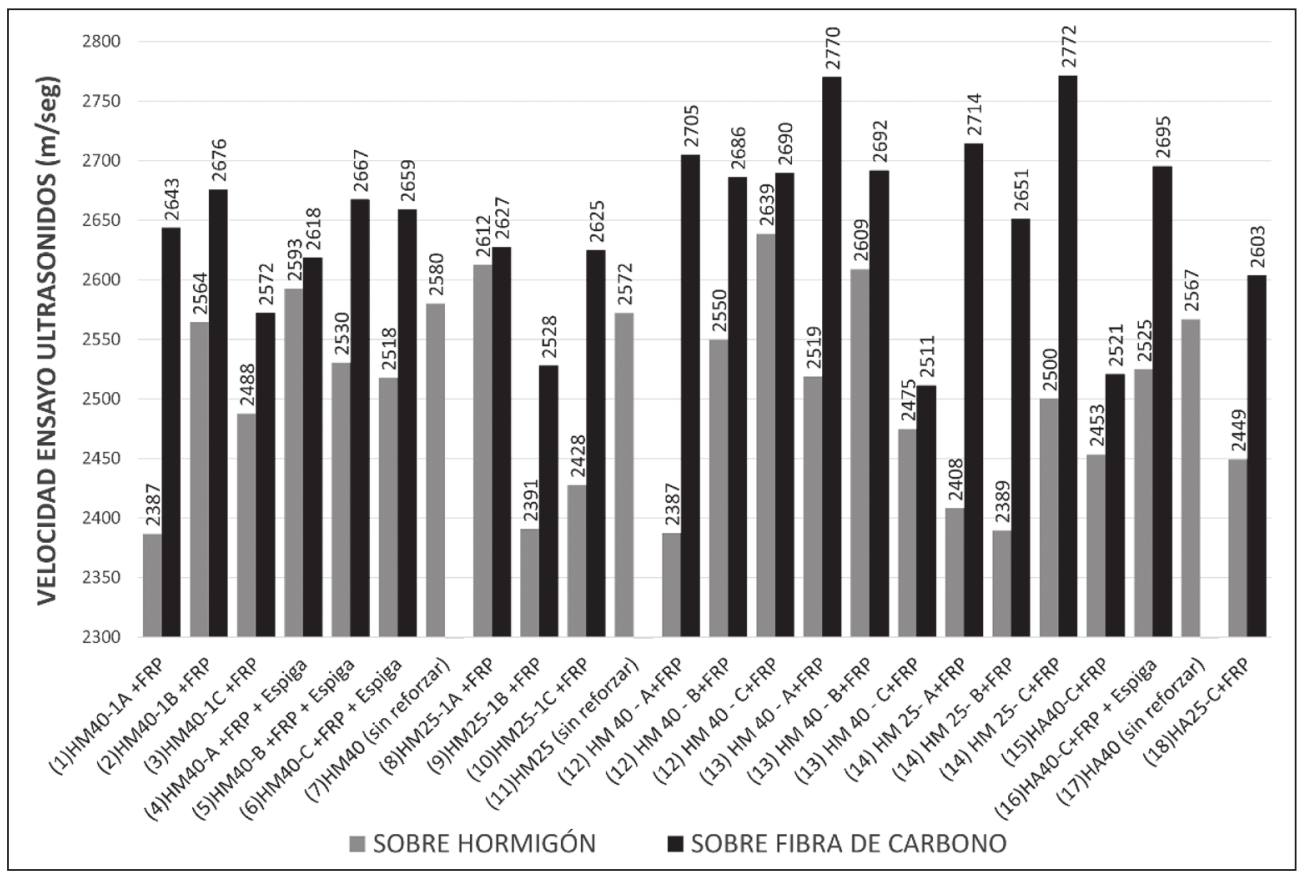

Fig.12. Resultados de ensayos de ultrasonidos antes y después de colocar el refuerzo Fuente: elaboración propia. 


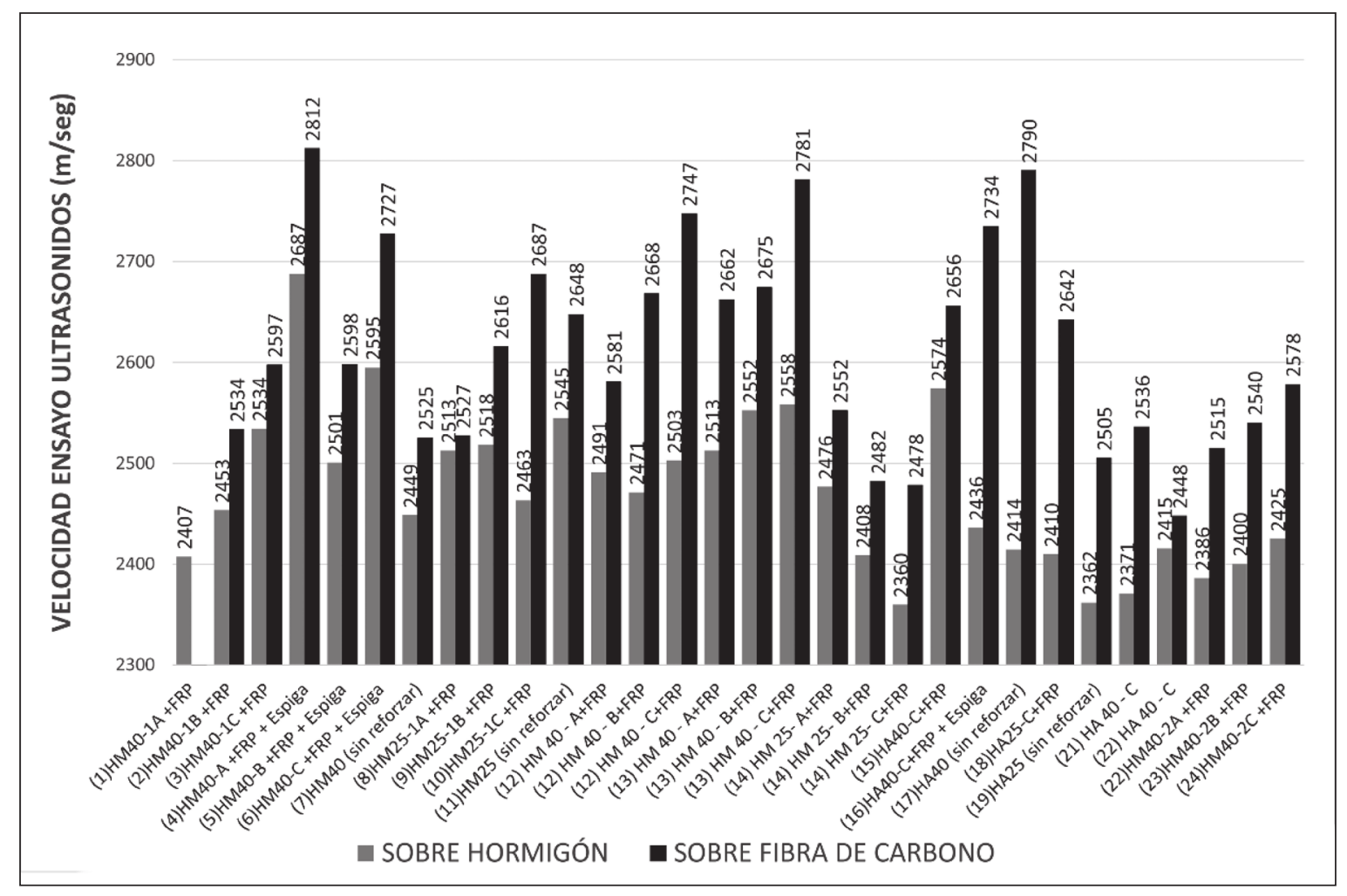

Fig. 13. Resultados de ensayos de ultrasonidos antes y después de colocar el refuerzo Fuente: elaboración propia.

En la Fig. 14 se observa una disminución de la velocidad de propagación para el tipo de concreto HM-40 con el aumento de la rugosidad; la velocidad para la rugosidad tipo $\mathrm{A}$ es de $2,710 \mathrm{~m} / \mathrm{s}$, mientras que para la rugosidad tipo $\mathrm{C}$ es de $2,617 \mathrm{~m} / \mathrm{s}$, esto es una disminución del $3,4 \%$. Sin embargo, para el tipo de concreto HM-25, aunque también existe una disminución de la velocidad de propagación de la rugosidad tipo A hacia la rugosidad tipo $B$, se produce una mejora de la velocidad en la rugosidad tipo $\mathrm{C}$. Este comportamiento para las probetas de concreto HM-25 puede ser debido a la mayor porosidad que tiene un concreto de menor resistencia. 


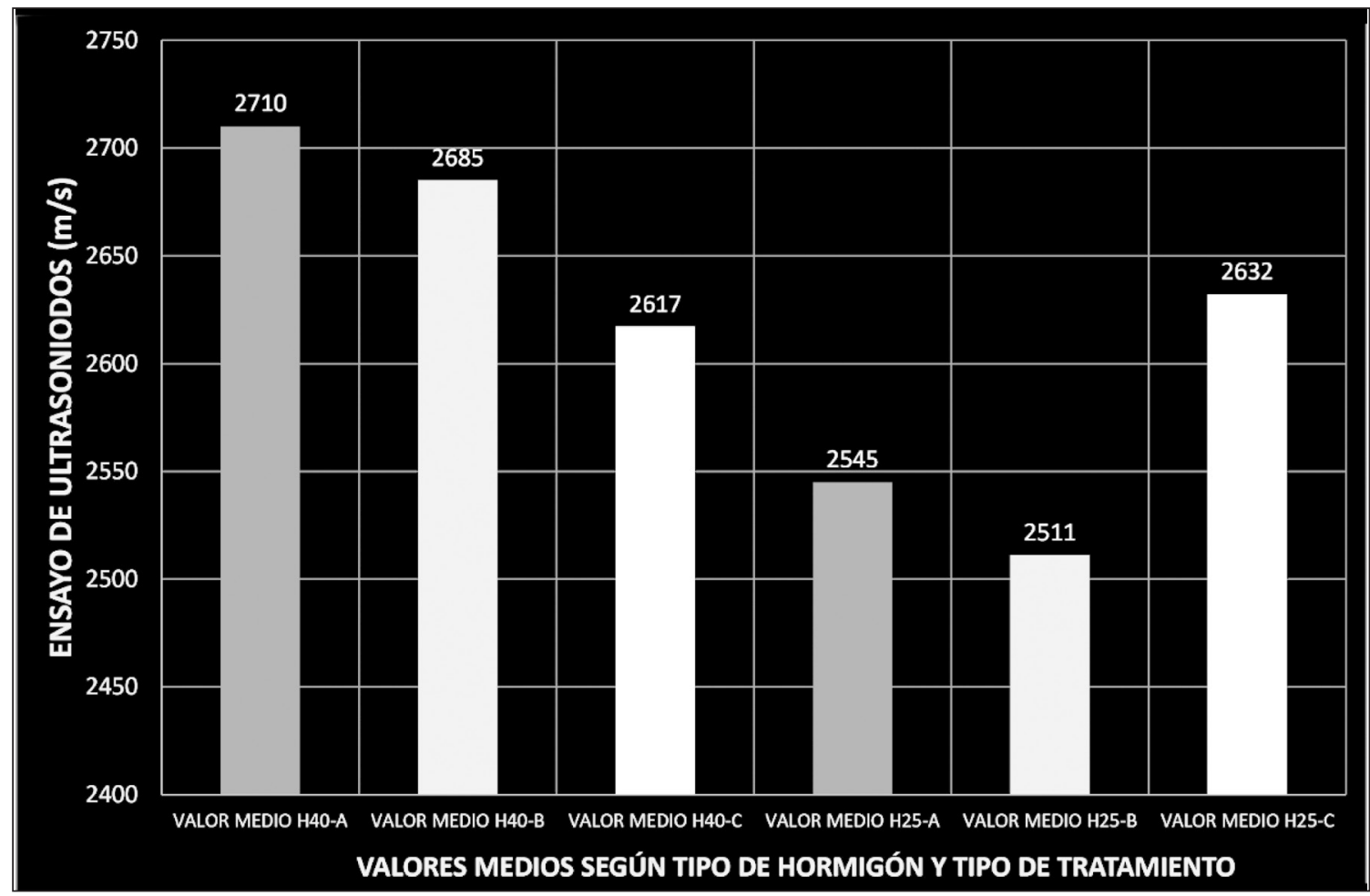

Fig. 14. Resultados medios ultrasonidos según tratamiento superficial y tipo de concreto Fuente: elaboración propia.

\subsection{Resultados ensayos de flexión}

Se analizaron probetas de concreto en masa y probetas armadas según cuantías previstas, con y sin refuerzo de FRP. En la Fig. 15 se presenta el valor de resistencia a flexión en MPa del ensayo de flexión para los tipos de probeta ensayadas en función del tipo de concreto y refuerzo con FRP.

Para el tipo de concreto HM-40, el mayor valor de resistencia a flexión se obtiene para la preparación de la superficie media (tipo B) 8,69 MPa, pero no existe una gran variación de la resistencia a flexión entre las tres preparaciones superficiales, que muestran valores respectivos para $A, B$ y $C$ de $8,41 \mathrm{MPa}$, $8,69 \mathrm{MPa}$ y $8,13 \mathrm{MPa}$.

Por lo que respecta al concreto tipo HM-25, existe claramente un valor de mejora considerable de la resistencia a flexión para la preparación de la superficie tipo $C$ (Fig. 16), de 7,69 $\mathrm{MPa}$ en la tipo $A, a$ 8,64 MPa en la tipo $C$. 
APLICACIÓN DE LAS TÉCNICAS NO DESTRUCTIVAS PULL-OFF Y ULTRASONIDOS EN EL CONTROL DE CALIDAD DEL REFUERZO CON MATERIALES COMPUESTOS EN ESTRUCTURAS DE CONCRETO

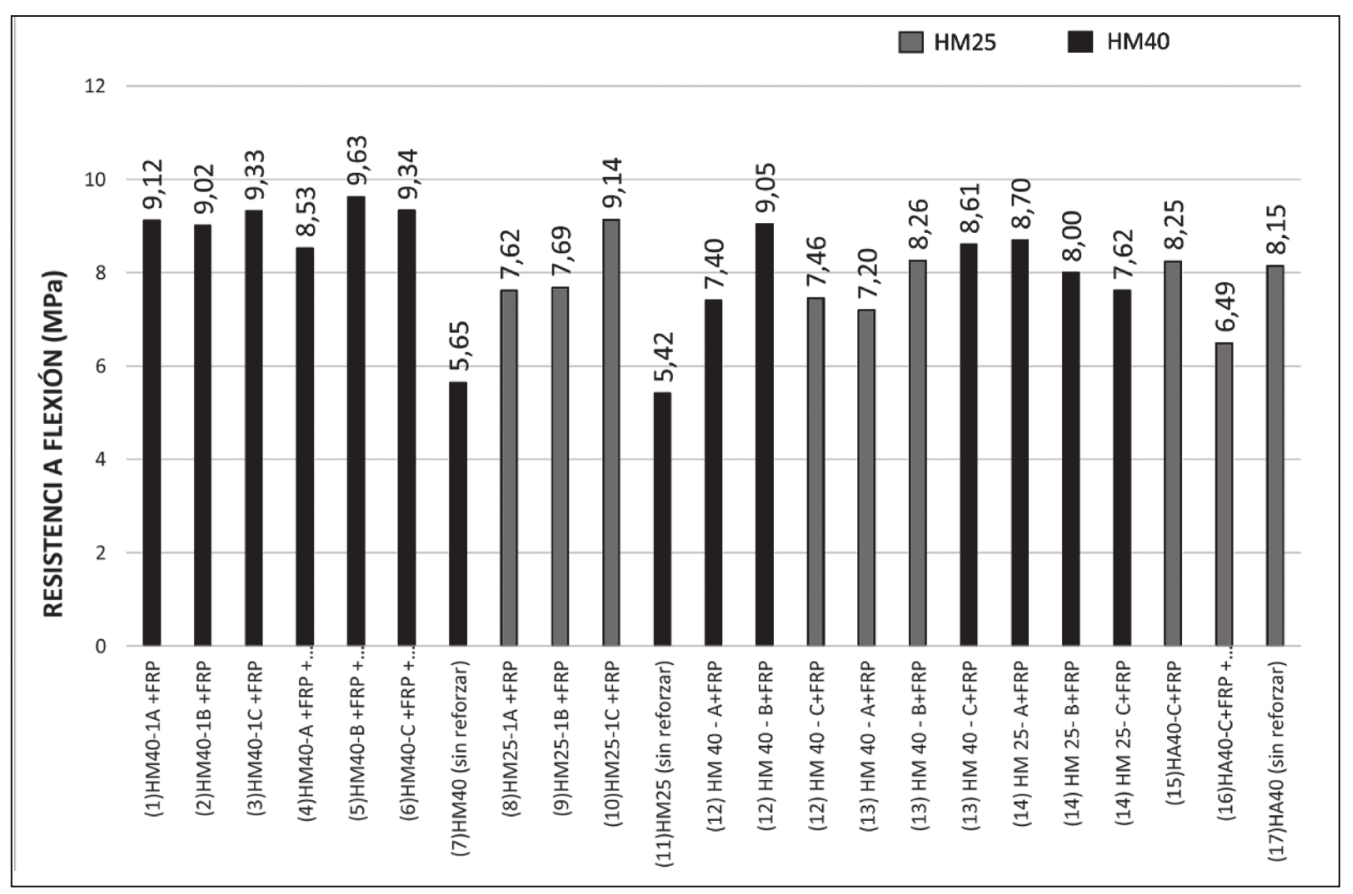

Fig. 15. Resultados de ensayos a flexión realizados Fuente: elaboración propia.

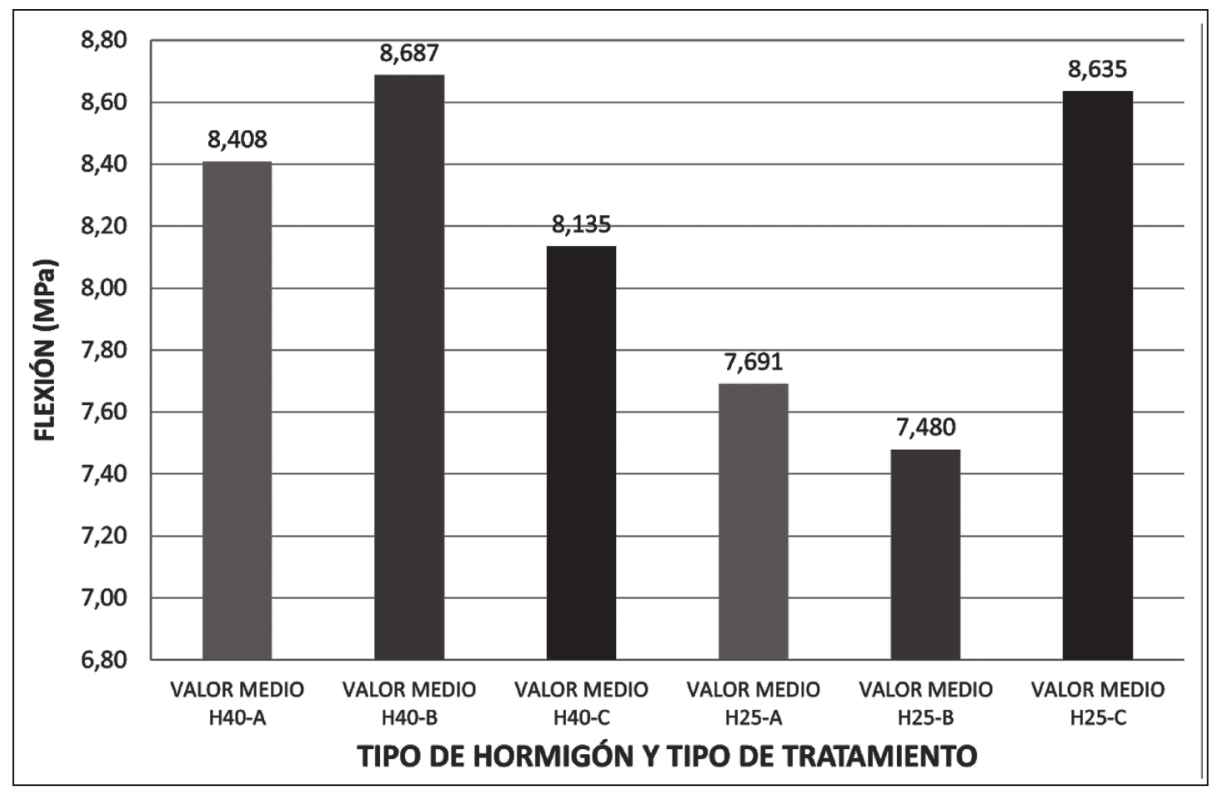

Fig.16. Resultados de rotura a flexión según tratamiento superficial y tipo de concreto Fuente: elaboración propia. 
Una vez analizados los resultados obtenidos de cada uno de los ensayos con respecto al tipo de concreto y las distintas preparaciones de la superficie para la adhesión de las bandas de FRP, y teniendo en cuenta el objetivo de establecer un método de control de calidad del refuerzo fiable y no destructivo, se han analizado posibles combinaciones de técnicas de ensayo, y se ha establecido de esta manera una relación entre los resultados alcanzados en los ensayos de ultrasonidos y los ensayos tipo pull-off.

En el diseño del plan de control de calidad sobre refuerzos de FRP en obra, es preciso considerar ensayos que sean de fácil ejecución, ya que los elementos reforzados pueden tener un acceso dificultoso.

De los ensayos realizados en la investigación, el de ultrasonidos es el método más adecuado, porque al tratarse de un ensayo no destructivo puede ser empleado en mayor extensión. Si con este ensayo podemos establecer parámetros de calidad de la adhesión del refuerzo de FRP y, por ende, del aumento de la capacidad mecánica del refuerzo, se podrá definir un método de control de calidad rápido, no destructivo y válido para evaluar la calidad del refuerzo ejecutado.

A continuación, se muestra la relación entre la velocidad en el ensayo de ultrasonidos y la tensión de arrancamiento que provoca la rotura en los ensayos tipo pull-off, como ensayos de control de calidad in situ.

\subsubsection{Correlación entre los ultrasonidos y el ensayo de pull-off}

Previo a la ejecución de la posible correlación entre ensayos, se ha procedido a realizar un análisis estadístico de los resultados de la tensión de arrancamiento de los ensayos de pull-off al objeto de la validación de estos. Los resultados obtenidos se recogen en la Fig. 17.

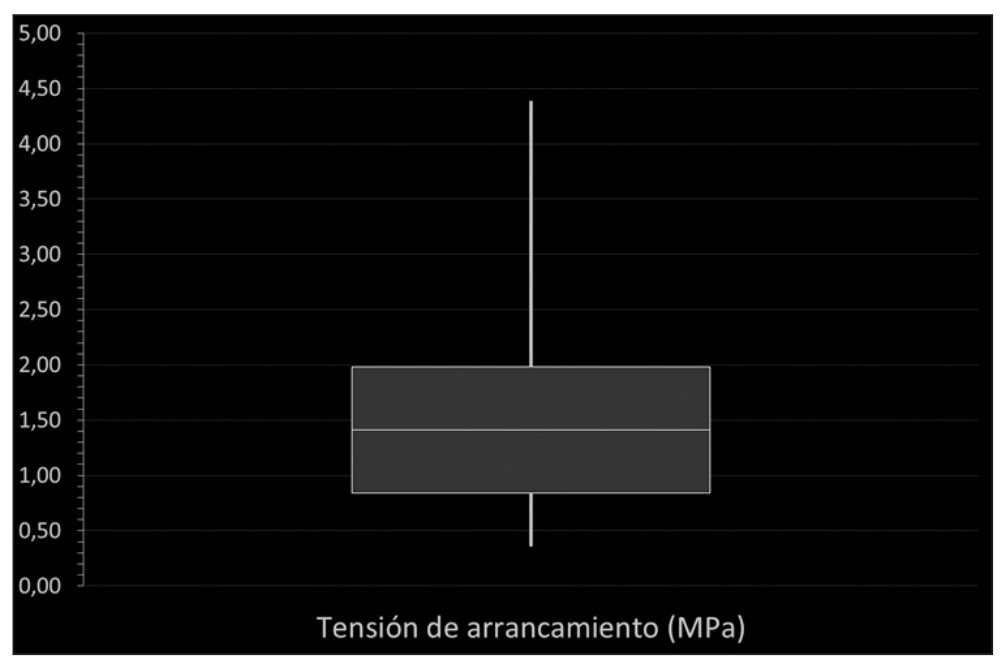

Fig. 17. Distribución de desviaciones de ensayos de pull-off Fuente: elaboración propia. 
Desechando los valores erróneos del ensayo de pull-off, se efectúa un análisis entre los resultados obtenidos en los dos ensayos, sin distinción por tipo de concreto, y se observa que existe una correlación entre estos, con un coeficiente de confianza aceptable. En la
Fig. 18 se presentan los valores conseguidos con dos tipos de regresiones (lineal y polinómica).

Asimismo, en la Fig. 19 se recoge la correlación y las regresiones de sendos ensayos, y se distinguen por tipo de concreto.

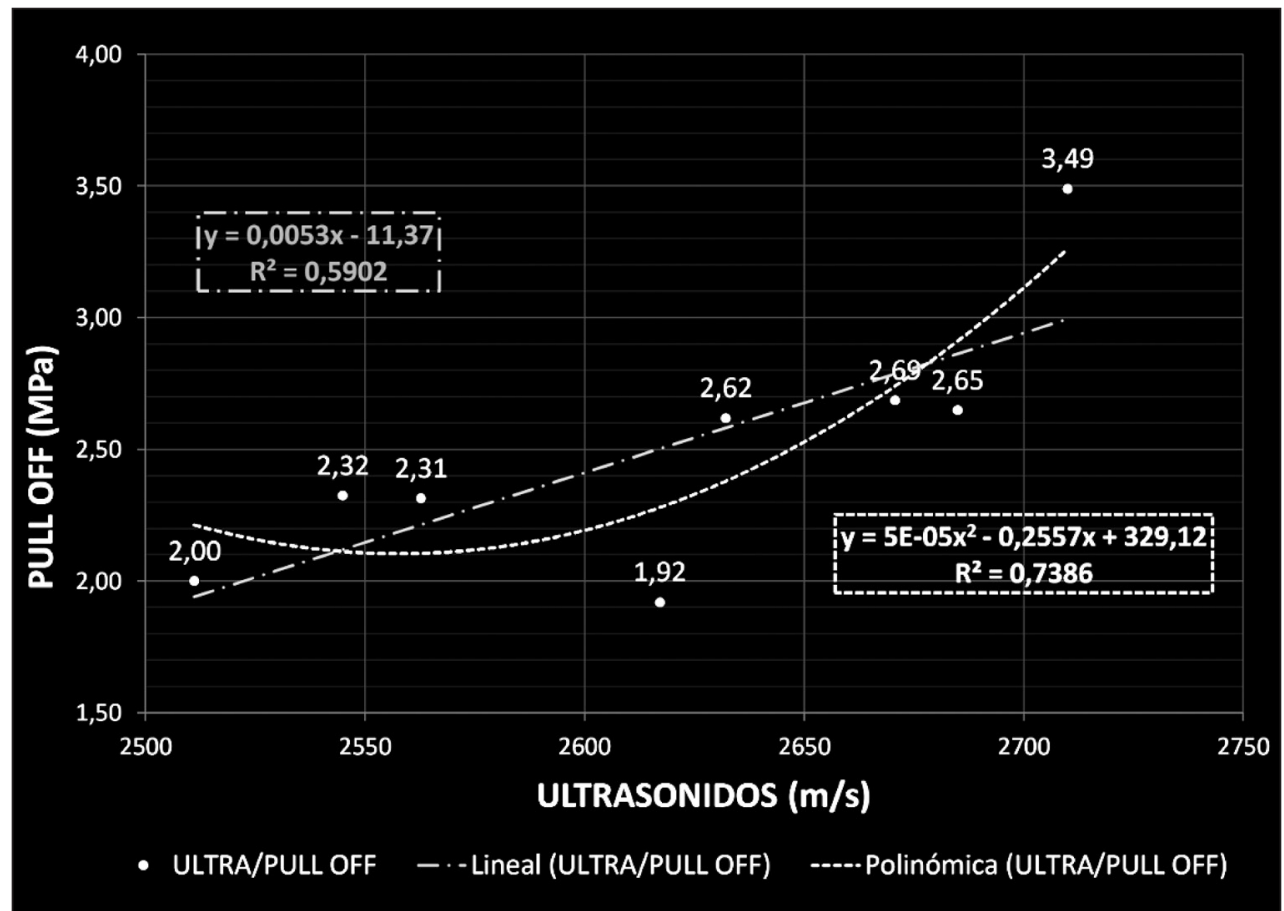

Fig.18. Resultados generales de pull-off y ultrasonidos Fuente: elaboración propia. 


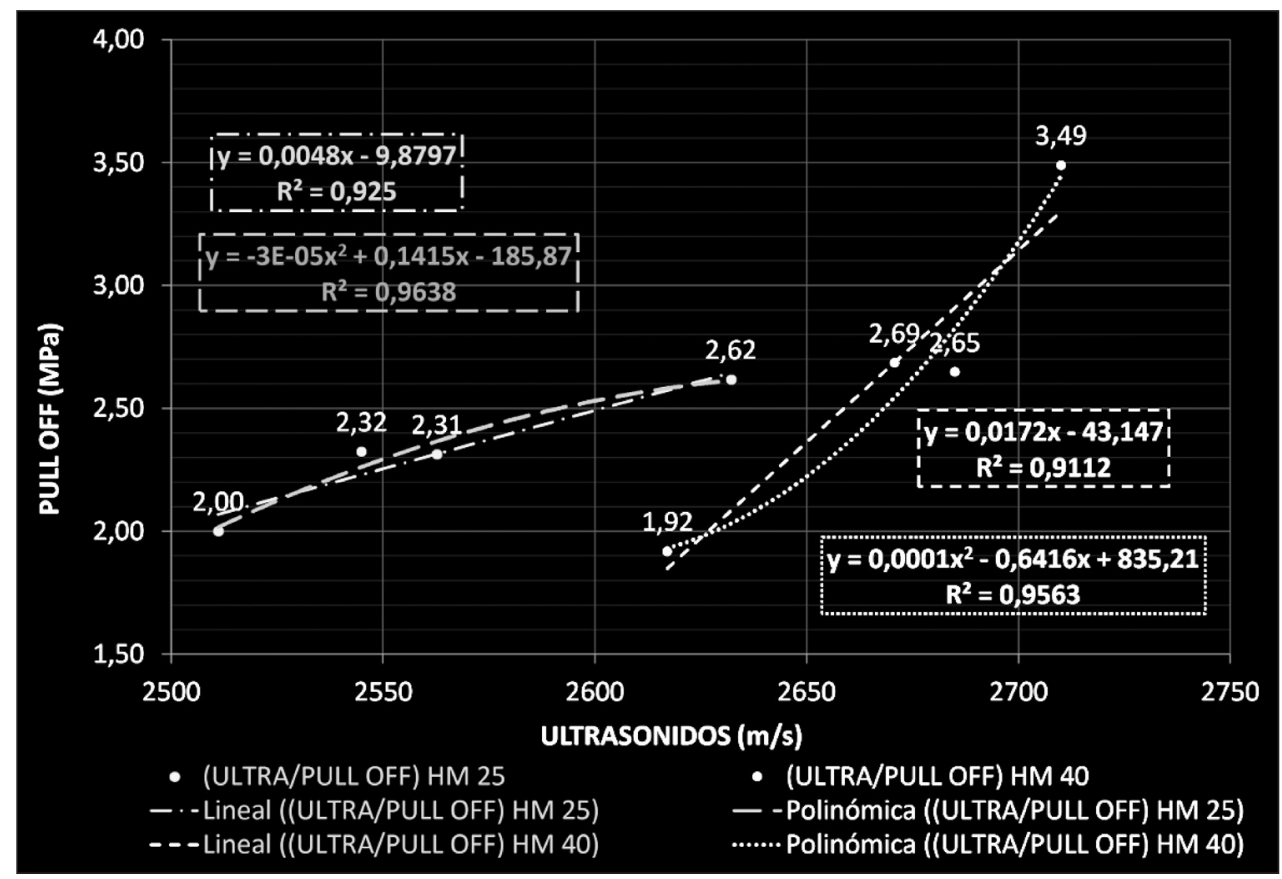

Fig. 19. Resultados de pull-off y ultrasonidos por tipo de hormigón Fuente: elaboración propia.

Ante la mayor facilidad, y aun presentando menor coeficiente de confianza, en este trabajo se propone una relación lineal entre velocidad de ultrasonidos y resistencia a la tracción de adherencia del FRP, como método de ensayo de control no destructivo para valorar la calidad del refuerzo. Según los resultados alcanzados, se presentan las siguientes ecuaciones, para el caso de regresión lineal.

Concreto HM40: $\quad y=0,0172 x-43,147$

Concreto HM25: $\quad \boldsymbol{y}=0,0048 \mathrm{x}-9,8797$

Ambos tipo de Concreto: $y=0,0053 x-11,37$ (3)

\subsubsection{Correlación entre resistencia a flexión y el ensayo de pull-off}

En este estudio se aalizan también los resultados de los ensayos de flexión frente a los resultados de pull-off, con el objetivo de validar la correlación anterior. En la Fig. 20 se constata la correlación entre la resistencia a flexión de las probetas y los resultados de los ensayos de Pull-off, como se puede observar, existe una correlación entre los resultados de los dos ensayos para las probetas ensayadas. Sin embargo, no se recomienda tomar esta correlación como un método válido para cuantificar numéricamente la mejora del comportamiento a flexión de elemento reforzado mediante los ensayos de puII-off, pero sí para demostrar la validez de estos ensayos como sistema de control de calidad. 


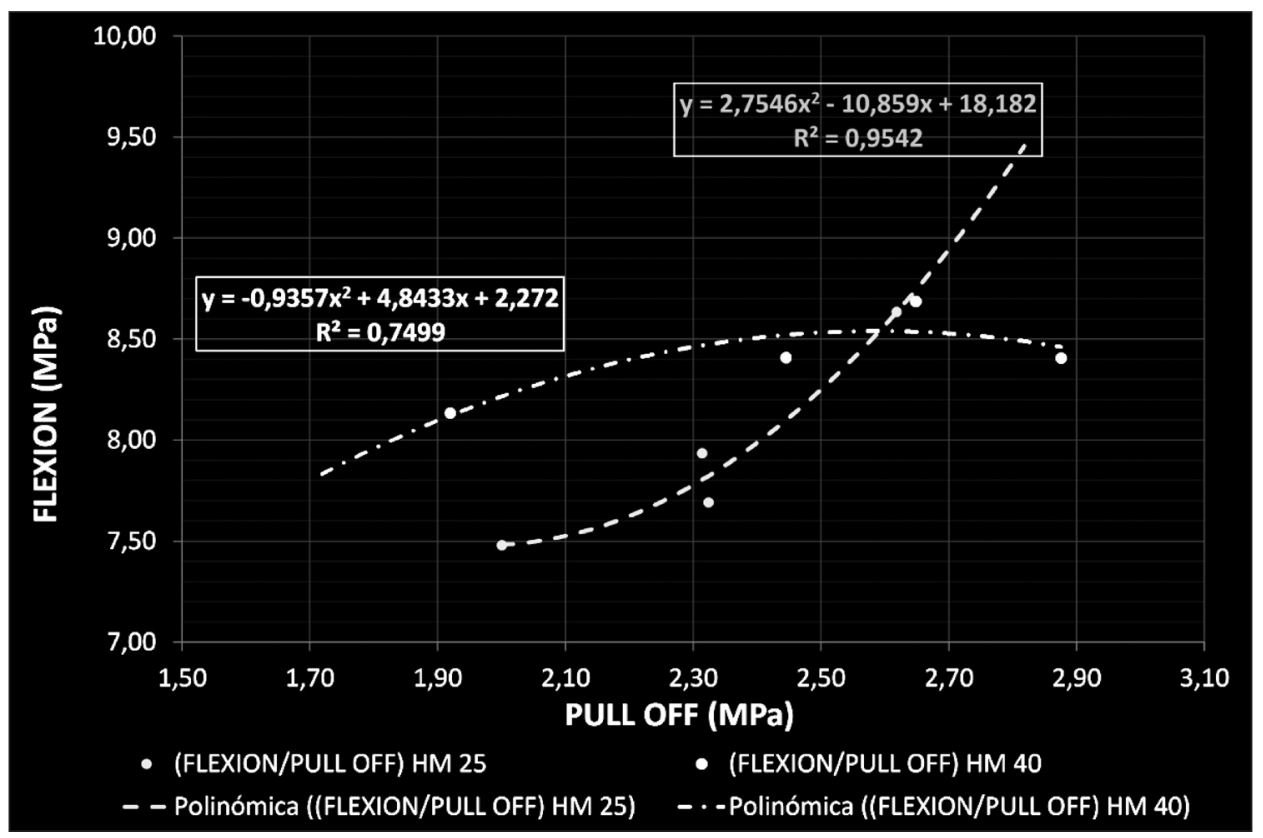

Fig. 20. Resultados de rotura a flexión según tipo de Concreto y pull-off Fuente: elaboración propia.

\section{CONCLUSIONES}

El control de calidad de las obras de refuerzo de estructuras de concreto armado con materiales compuestos FRP debe incluir ensayos para evaluar la ejecución, como los pull-off y ultrasonidos.

Mediante ensayos pull-off es posible determinar la preparación del soporte más óptima en función del tipo de concreto y validar la calidad del refuerzo. En la investigación efectuada se ha comprobado que en el concreto de mayor resistencia la preparación del soporte no incide en la mejora de la adherencia de las bandas de refuerzo. Mediante la ejecución de ensayos de ultrasonidos es posible verificar la preparación del soporte o rugosidad de la superficie, sobre todo en concretos de alta resistencia.
De igual forma, los ensayos de ultrasonidos se pueden emplear para verificar la adecuada preparación del soporte o rugosidad de la superficie, sobre todo en concretos de alta resistencia. En la investigación realizada se demuestra que existe una correlación entre la velocidad de propagación de ultrasonidos y la tensión de arrancamiento en los ensayos pull-off, y a su vez este último se correlaciona con el comportamiento a flexión de refuerzo. Por tanto, mediante el empleo de un ensayo no destructivo, como son los ultrasonidos, es posible valorar el comportamiento del refuerzo.

La valoración cualitativa con un estudio extensivo mediante ultrasonidos, en gran parte de la superficie de los elementos reforzados y complementada con una serie de ensayo de pull-off seleccionados en función de los ultrasonidos, permitiría cuantificar el 
comportamiento del refuerzo. Si bien, no se considera adecuado establecer correlaciones generales según el tipo de concreto, es recomendable realizar dicha correlación en cada refuerzo por verificar. Este aspecto vale la pena considerarlo en nuevas investigaciones, para incorporar así otro tipo de ensayos de posible ejecución in situ en las obras.

Por tanto, y como conclusión final, es posible efectuar un control de calidad del refuerzo de estructuras de concreto armado mediante FRP con ensayos no destructivos como ultrasonidos y pull-off, y al mismo tiempo se recomienda su inclusión en los protocolos de inspección. Así pues, como ha quedado demostrado, la variación transmisión de la onda ultrasónica a través del refuerzo puede estar relacionada con la diferente adherencia de este, valorada a través de los ensayos de pull-off.

\section{AGRADECIMIENTOS}

La investigación se inició en el ámbito del proyecto Nuremco (proyecto $n^{0}$ : ITC-20131020), financiado por el Ministerio de Economía y Competitividad, dentro del denominado programa Feder Innterconecta en 2013-2015, con cargo al Fondo Tecnológico (fondos Feder) (Programa Operativo de I+D+i por y para el beneficio de las empresas, Fondo Tecnológico); posteriormente lo continuaron miembros del grupo de investigación Paidi TEP954, de la Escuela de Arquitectura de Sevilla y la empresa Labrum S.L.

\section{REFERENCIAS}

[1] W. R. Escobar, H. B. Barrera y H. V. Amarís, "Importancia estratégica de los nuevos materiales en el desarrollo sostenible y como alternativa de competitividad," Revista Ciencia e Ingeniería Neogranadina, vol. 8, pp. 33-42, 1999.

[2] A. Besednjak y A. B. Dietrich. Materiales compuestos. Barcelona, España: Universidad Politécnica de Cataluña, 2009.

[3] M. Olivares Santiago, C. Galán Marín y J. Roa Fernández, "Los Composites. Características y aplicaciones en la edificación," Informes de la Construcción," vol. 54, no. 484, pp. 45-62, 2003. DOI: https://dx.doi.org/10.3989/ ic.2003.v54.i484.568.

[4] C. E. Bakis, A. Ganjehlou, D. I. Kachlakev, M. Schupack et al. (2002). Guide for the design and construction of externally bonded FRP systems for strengthening concrete structures. $\mathrm{ACl}$ Committee, 440. Michigan, Estados Unidos: American Concrete Institute.

[5] T. Horiguchi y N. Saeki, "Effect of Test Methods and Quality of Concrete on Bond Strength of CFRP Sheet. Non-Metallic (FRP) Reinforcement for Concrete Structures," en 3th. Int. Symp. Japan Concrete Institute, 1997, pp. 265-270.

[6] D. G. Allen y R. A. Atadero, "Evaluating the long-term durability of externally bonded FRP via field assessments," Journal of Composites for Construction, vol. 16, no. 6, pp. 737-746, 2012. DOI: https://doi.org/10.1061/ (ASCE)CC.1943-5614.0000305

[7] Y. Bai, P. A. M. Basheer, D. J. Cleland y A. E. Long, "State-of-the-art applications of the pull-off test in civil engineering," International Journal of Structural Engineering, vol. 1, no. 1, 
pp. 93-10, 2009. DOI: https://doi. org/10.1504/ijstructe.2009.030028

[8] ASTM D7522. Standard Test Method for Pull-Off Strength for FRP Laminate Systems Bonded to Concrete Substrate, ASTM International, West Conshohocken, PA, 2015. DOI: https://doi. org/10.1520/D7522_D7522M-15

[9] T. Alkhrdaji, E. R. Fyfe, V. M. Karbhari, M. Schupack et al. 440.3R-12 Guide Test Methods for Fiber-Reinforced Polymers (FRPs) for Reinforcing or Strengthening Concrete Structures. Michigan, Estados Unidos: American Concrete Institute, 2004.

[10] UNE-EN 1542. Productos y sistemas para la protección y reparación de estructuras de hormigón. Métodos de ensayo. Determinación de la adhesión por tracción directa. Madrid, España: Aenor, 2000.

[11] C. Scarponi y G. Briotti, "Ultrasonic technique for the evaluation of delaminations on CFRP, GFRP, KFRP composite materials," Composites Part B: Engineering, vol. 31, no. 3, pp. 237-243, 2000. DOI: https://dx.doi.org/10.1016/ S1359-8368(99)00076-1

[12] F. Bastianini, A. Di Tommaso y G. Pascale, "Ultrasonic non-destructive assessment of bonding defects in composite structural strengthenings," Composite Structures, vol. 53, no. 4, pp. 463-467, 2001.

[13] M. Ekenel y J. J. Myers, "Nondestructive evaluation of RC structures strengthened with FRP laminates containing near-surface defects in the form of delaminations," Science and Engineering of Composite Materials, vol. 14, no. 4, pp. 299-315, 2007.
[14] R. Helmerich, B. Milmann y J. Wöstmann, "Non-destructive detection of surface-bond defects in carbon composite-strengthened concrete structures," Structure and Infrastructure Engineering, vol. 11, no. 1, pp. 3-14, 2015. DOI: https://doi.org/10.1080/1573247 9.2013 .879322

[15] M. Ekenel y J. J. Myers, “Durability performance of RC beams strengthened with epoxy injection and CFRP fabrics," Construction and Building Materials, vol. 21, no. 6, pp. 1182-1190, 2007. DOI: http://dx.doi.org/10.1016/j. conbuildmat.2006.06.020

[16] A. De Diego, A. Arteaga, J. Fernández, R. Perera y D. Cineros, "Behaviour of FRP confined concrete in square columns," Materiales de Construcción, vol. 65, no. 320, pp. 1-9, 2015. DOI: http://doi. org/10.3989/mc.2015.05414

[17] J. Szymanowski y Ł. Sadowski, "Ultrasonic Pulse Velocity Evaluation of the Pull-Off Adhesion between Epoxy Resin and Concrete Substrate," en Key Engineering Materials (vol. 728) Trans Tech Publications, Polonia, Wroclaw University of Sccience and Technology, 2017, pp. 390-395.

[18] UNE-EN 12390-5. Ensayos de hormigón endurecido. Parte 5: Resistencia a flexión de probetas. Madrid, España: Aenor, 2005.

[19] UNE-EN 12504-4. Ensayos de hormigón en estructuras. Parte 4: Determinación de la velocidad de los impulsos ultrasónicos. Madrid, España: Aenor, 2006. 